\title{
INTERPRETACIÓN DE SISTEMAS NATURALES COMPLEJOS: EL PROBLEMA DE LOS TAFONI
}

\author{
Elena de Uña Álvarez \\ Departamento de Historia, Arte y Geografía. Universidad de Vigo \\ edeuna@uvigo.es
}

\section{RESUMEN}

La interpretación de las formas del relieve integra la diferenciación de componentes en el paisaje, su categorización mediante propiedades genéricas y su conexión con procesos complejos en el espacio-tiempo. Conceptos y categorías utilizados en la investigación de los tafoni revelan diversos planteamientos en el marco de la actividad científica. El objetivo del artículo es analizar la trayectoria de la interpretación de estas formas y los problemas planteados. Considera las evidencias del trabajo de campo, la articulación de conceptos y la relación entre diversos significados de un complejo ligado a fenómenos naturales irreversibles.

Palabras clave: Tafoni, sistema natural, forma de relieve, complejidad.

\begin{abstract}
Interpreting landforms implies to differentiate landscape components, their categorizations through generic properties, and their connection with processes which are complex in spacetime. Concepts and categories used to research tafoni forms reveal different approaches in the scientific field. This article aims to analyse the trajectory of the tafoni' interpretation and the related problems. Field research evidences, concepts articulation, and the relationship between different meanings of a complex linked to natural irreversible phenomena are to be considered.
\end{abstract}

Key words: Tafoni, natural system, landform, complexity.

Fecha de recepción: marzo 2011.

Fecha de aceptación: julio 2012. 


\section{INTRODUCCIÓN}

La interpretación de las formas del relieve ha sido tratada desde perspectivas históricas (Chorley et al., 1964; Kennedy, 2006) y epistemológicas (Scheidegger, 1961; Reynaud, 1971; Gutiérrez y Peña, 1988; López Bermúdez, 2002). Muestran la controversia entre orientaciones del conocimiento durante la mayor parte del siglo XX (Summerfield, 2005). La teoría general de sistemas reconsidera principios y métodos (Chorley, 1962; Chorley y Kennedy, 1971) impulsando los enfoques integrados. En este contexto, cualquier forma del relieve es un sistema natural en continuo cambio. Este concepto es fundamental en la elaboración de teorías (Martínez de Pisón, 1975; Thorn, 1988; Rhoads y Thorn, 1996; Inkpen, 2005) y en la selección de métodos de investigación (Tricart, 1965; Gregory, 1985; Haines-Young y Petch, 1986; Muñoz Jiménez, 1992; Church, 2010).

Las formas menores del relieve constituyen sistemas naturales identificados por tamaños y rasgos particulares. Las interpretaciones sobre su origen y desarrollo perpetúan o modifican su significado en la evolución del relieve, general o local. La definición de categorías a lo largo del tiempo expresa los resultados del análisis de la distribución espacial, la configuración morfológica y los posibles mecanismos genético-evolutivos. La búsqueda de su explicación científica se realiza en el marco de los enfoques del conocimiento geomorfológico.

Las formas menores denominadas tafoni presentan gran variedad de configuraciones morfológicas. Desde que fueron descritas por primera vez, hace más de un siglo, su interpretación es un problema y un reto para la investigación. La discusión sobre su origen y los fenómenos implicados en su evolución mantiene el interés de su estudio. Goudie (2004) las describe como huecos de dimensión métrica (esféricos o elipsoidales) con perfiles en arco y paredes internas cóncavas. Las cavidades (parcial o totalmente cerradas) crecen en la base de los bloques o lajas y en las superficies rocosas inclinadas; las paredes internas están diversificadas en ocasiones por micro-modelados cóncavos o convexos (Twidale y Vidal Romaní, 2005). Son frecuentes en granitos y areniscas, con dimensiones decimétricas-métricas y secciones circulares o elípticas (Gutiérrez Elorza, 2009): el fondo puede estar cubierto de detritos (sujetos a la acción del viento, lluvia o escorrentía) y su origen ha sido relacionado con mútiples procesos (erosión eólica, ciclos de humectación-secado, haloclastia, termoclastia, crioclastia, disolución de cementos carbonatados, meteorización subedáfica). Existe consenso sobre la relación de estas formas con el avance de la desagregación granular o de la descamación de la roca, al menos durante cientos de miles de años (Gregory y Goudie, 2011).

Al abordar el problema de su interpretación, partimos de las claves utilizadas para tratar de resolverlo en diversos escenarios. Los elementos de su configuración, asociados en principio a los procesos subaéreos. La diferenciación en categorías, relacionada con fenómenos proceso-respuesta en ambientes epigénicos. La diversidad morfológica, ligada a la dinámica no lineal de crecimiento. La complejidad de su desarrollo, con secuencias de permanencia y de cambio. El enfoque atiende especialmente a la variabilidad que presentan en granitos y areniscas. El análisis se centra en los enlaces entre las interpretaciones propuestas: la articulación de su concepto, la interacción de las evidencias y las hipótesis empleadas para explicar su comportamiento como un sistema natural. 


\section{CONFIGURACIÓN, PROCESO, RESPUESTA}

\section{Escenarios del descubrimiento}

En 1864 Casiano de Prado describió en el granito de la Sierra de Guadarrama (Madrid, Sistema Central Ibérico) «un horado en una masa saliente de granito taladrada de parte a parte...y aunque aquí la descomposición obra por todas partes es más fuerte en el centro que los costados» (Prado, 1976: 110) semejante a los foracos en las calizas de la Cordillera Cantábrica. Reusch (1882) designó los huecos internos en las rocas cristalinas de Córcega con el término local de tafone (ventana, en plural tafoni) por su apertura al exterior a través de una perforación, difundido por los estudios de Penck (1894). Estos trabajos son las referencias de Choffat (1895) para definir las cavidades, en la base de los bolos graníticos del norte de Portugal, como formas de erosión atmosférica. Hult (1899) relató las propiedades de formas similares en su trabajo sobre la alteración del granito en el macizo de Ourense (Galicia, noroeste del Macizo Ibérico).

Las primeras interpretaciones partieron de las cualidades macroscópicas (diferencias de tamaño, aspecto morfológico) y la presencia de productos de meteorización (escamas, granos, costras); en un tipo de roca y de ambiente natural, un fenómeno causa-efecto. El origen

\section{CATEGORIAS NOMINALES}

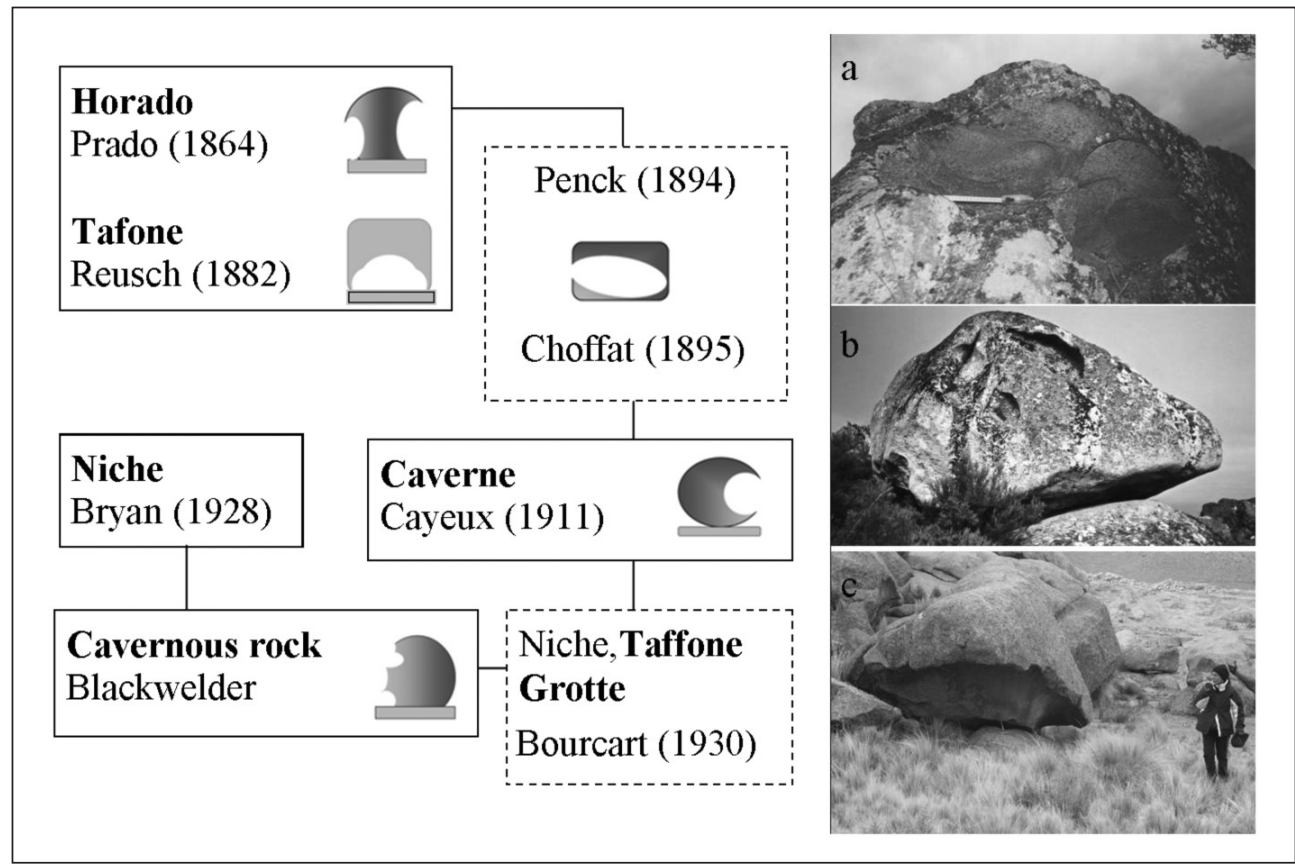

Fotografías: a) Montealegre, Ourense (primer tafone descrito por Hult en Galicia); b) Serra de Gomariz, Baltar, Ourense (Galicia, noroeste del Macizo Ibérico); c) Los Riojanos, Córdoba (noroeste de las Sierras Pampeanas, Argentina). 
de las formas se atribuyó a la acción del viento y la cristalización de sales (Futterer, 1902; Cayeux, 1911; Hume, 1925), a la erosión diferencial de los materiales bajo una capa dura formada por deposición de hierro o sílice (Jutson, 1917) y a la concentración de la humedad en áreas de sombra (Passarge, 1920). Bryan (1928) aludió a la disolución para los casos en areniscas de Arizona y Nuevo México (EE.UU.). Blackwelder (1929), a ciclos de humectación-desecación para los de los granitos en el desierto de California (EE.UU.) con un crecimiento en dos etapas (meteorización y erosión). Desde entonces, el uso del término general caverne coexiste con los específicos de tafone, cavernous rock y niche (Fig. 1). La definición universal incluye algunos rasgos morfológicos típicos; en realidad designan un cortejo de formas que denominaremos tafoni.

\section{Condiciones de estado}

La definición posterior de categorías morfológicas utiliza el criterio de la posición del plano de apertura de la cavidad (Fig. 2). El estado de las formas resulta de las condiciones del medio climático, con posibles situaciones de inversión funcional (Birot, 1958). Su interpretación se apoya en las nociones de sistema de erosión y de dominio morfoclimático (Cholley, 1950; Tricart y Cailleux, 1965).

En los dominios áridos/semiáridos algunos autores proponen que la desagregación granular y la descamación tienen su origen en la expansión-contracción de la roca por termoclastia (Kvelberg y Popoff, 1938) o crioclastia (Cailleux, 1953; Klaer, 1956). En los dominios glaciares, por la crioclastia (Nichols, 1953) y la acción erosiva de los cristales de hielo movilizados por el viento (Voronov, 1958). La mayoría reafirman un origen por haloclastia (Evans, 1969) sobre todo en medios litorales áridos/semiáridos (Prebble, 1967; Denaeyer, 1953; Segestrom y Henríquez, 1964). La formación de capas duras, debido a la precipitación de hidróxidos de hierro en superficies expuestas a la insolación y la meteorización química debido a la concentración de la humedad en la base de las rocas vuelven a mencionarse como factores de la erosión diferencial (Wilhelmy, 1958).

$\mathrm{Si}$ el concepto es el de una forma diferenciada bajo ciertas condiciones ambientales, su análisis en el contexto de la evolución del clima permitirá reconocer estados fósiles y activos. Para Tricart (1952), la mayoría de los estados en los granitos de Córcega son relictos, ligados a procesos antiguos de meteorización química y de erosión (dirigidos por la red de diaclasas). Ottmann (1956) distingue en el mismo lugar las formas fósiles, con paredes internas colonizadas por líquenes, de las formas activas, con paredes internas que crecen por el progreso de la descamación. En las granodioritas del desierto de Chile, Grenier (1968) caracteriza los estados menos evolucionados de formas activas por la descamación intensa en la cavidad interna, el mantenimiento casi intacto de su techo (bóveda) y la conservación de una película ferruginosa en su superficie externa.

Según Wilhelmy (1964), aunque a cada clima corresponde un tipo particular de alteración, los fenómenos físicos y químicos (erosión eólica, hidratación, oxidación, disolución, humectación-desecación) actúan conjuntamente en la génesis de los tafoni; el predominio de un proceso específico varía en función de la escala de análisis (regional, local, estacional). Con esta perspectiva comienza el registro de las medidas (ejes de la cavidad) para estimar su edad relativa. La interpretación como un sistema natural proceso-respuesta, contempla diver- 


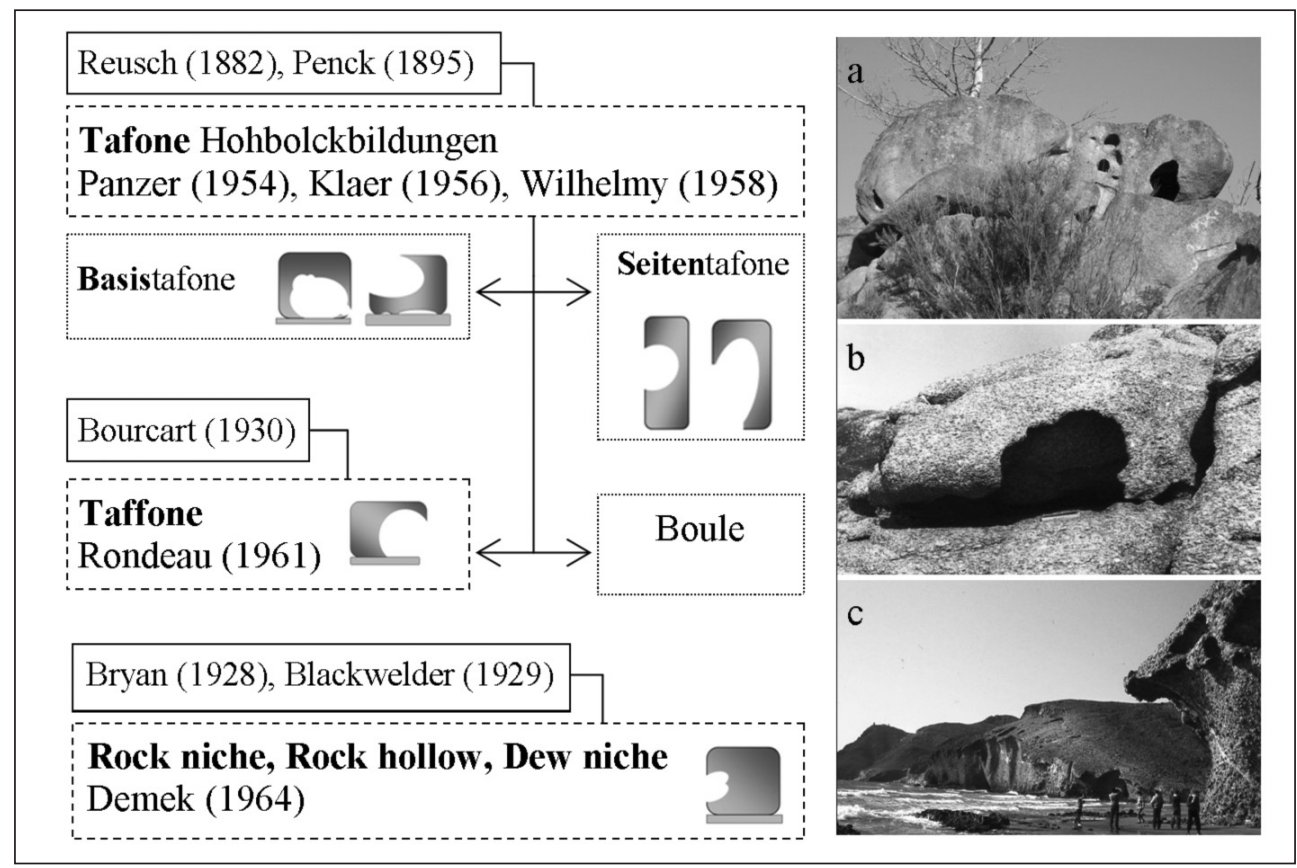

Fotografías: a) Replano de Trasalba, Ourense (Galicia); b) Monte dos Castelos, A Coruña (Galicia); c) Cabo de Gata, Almería (Andalucía).

sos grados de ajuste respecto a las variaciones de su entorno - diversos tipos de equilibriodefinidos en función de la escala del análisis (Schumm y Lichty, 1965).

El trabajo de Calking y Cailleux (1962) en las morrenas del valle Victoria (Antártida) demostró la relación directa entre la edad de exposición subaérea de los bloques (gneiss, granito) y la profundidad del volumen excavado por las formas (en este supuesto de origen exógeno). Sin embargo, Dragovich (1969) afirmó que la localización y dimensiones en los granitos del sur de Australia resultan de secuencias de meteorización química subedáfica y de erosión post-pliocenas. El análisis de Martini (1978) sobre las propiedades de una serie de casos (dimensiones, morfología interna, colonización biótica, microclima) en los conglomerados de Monte Pisani (Toscana, Italia) confirmó la intervención de diferentes procesos en el origen y la evolución de las formas (crioclastia, haloclastia, humectación-desecación, hidrólisis). Vidal Romaní et al. (1979) con los registros cualitativos y cuantitativos de las formas en el Monte Louro (Galicia) demostraron que, en los granitos, la superficie de partida es un plano de discontinuidad; la erosión de la roca progresa configurando una bóveda interna, cuya evolución está determinada por condiciones límite (relativas a la dinámica de crecimiento interno o a la estabilidad de los apoyos en un bloque).

Las monografías del momento sobre las formas de modelado definían condiciones de inicio, irregularidades en la textura o estructura de la roca, y de crecimiento, procesos asocia- 
dos a los dominios climáticos (Mainguet, 1972; Godard, 1977). Sin embargo, el estudio de procesos y respuestas constataba el carácter ambiguo de categorías morfológicas asociadas al clima. De acuerdo con Martini (1978 o.c.), el problema en la interpretación de los tafoni era su concepto como resultado de un proceso y la dificultad de conocer su desarrollo, que integra varias fases de crecimiento y de ralentización.

\section{DIVERSIDAD, PERMANENCIA, CAMBIO}

\section{Naturaleza de la morfogénesis}

\subsection{Desarrollo polifásico}

La diversidad morfológica manifiesta una serie de transformaciones relacionadas con umbrales geomorfológicos (Fig. 3). Las evidencias sobre configuraciones semejantes en diferentes dominios climáticos (Tschang, 1974; Rögner, 1988; Kejonen et al, 1988) y tipos de roca (Teodoropoulos, 1974; Höllermann, 1975; Sanz, 1976; Smith, 1978; Kelletat, 1980) confirman su carácter azonal. Pero la explicación desde el enfoque proceso-forma plantea el problema de la equifinalidad (Haines-Young y Petch, 1983; Gerrard, 1984). El estudio de las interacciones de varios procesos, en materiales con propiedades mineralógicas y estructurales variadas, trata de resolverlo.

Al mismo tiempo, se articula el concepto de las formas de relieve como sistemas complejos. Su dinámica conecta fases de permanencia y de cambio. La herencia representa la condición inicial en un momento dado. Puede mantenerse o no durante la evolución, regulada por retroalimentaciones complejas. El estado de las formas es una función de las propiedades de la roca, los flujos energéticos, el clima y la actividad biótica (Brunsden y Thornes, 1979): la propagación del cambio tiene lugar de manera ubicua (por ejemplo, en los procesos de meteorización subedáficos), lineal (en su canalización a través de las diaclasas) o difusa (a través de las conexiones con otros sistemas geomorfológicos). La transición entre fases tiene lugar una vez que se alcanzan ciertos valores críticos (Thornes, 1983; Brunsden, 1996). La interpretación de la permanencia y del cambio considera una cascada de estados (Spedding, 1997). Al aplicar este planteamiento en la investigación de las formas, la clave del análisis es la variabilidad.

La morfogénesis de los tafoni funciona mediante bucles de retroalimentación positivos y negativos (Tricart y Cailleux, 1969) que replican, transforman o degradan su configuración. La composición de las rocas, su textura y estructura, y las variaciones microclimáticas entre el interior-exterior de la cavidad son variables de control en su dinámica. Determinan la capacidad de absorción del agua, un agente de primer orden en los procesos de meteorización y erosión. En areniscas, la morfogénesis se relaciona también con la localización de las formas, el contenido en matriz arcillosa o las costras de endurecimiento (Rodríguez y Navascués, 1982; Ugarte, 1983; Soriano, 1987). La mayoría de los investigadores señalan que las modificaciones periódicas de la humedad junto con la cristalización de sales son responsables de la génesis, dominando las primeras fases de crecimiento (Williams y Robinson, 1982; Sancho y Benito, 1990). La formación o la erosión de las costras salinas intervendrían en la modificación posterior de su tendencia, o en su bloqueo (Mustoe, 1983; 
Edeso, 1988). Los efectos de la crioclastia, la hidrólisis y la disolución de cementos dependen en gran medida de la intensidad de la hidratación y de la haloclastia (Sánz Pérez, 1996; Turkington, 1998). En los granitos, la distribución espacial y la geometría de las formas están asociadas en muchos casos a procesos de deformación mecánica, en el marco de un sistema de bloques o de un afloramiento (Vidal Romaní, 1985). La localización de cavidades en el contacto roca sana/alterada conecta su origen con procesos de meteorización subsuperficial (hidratación, hidrólisis) y la posterior erosión de sus productos (regolito) bajo control de la estructura; mientras que su apertura en la base de los bloques implica una estrecha relación con la meteorización asociada al movimiento del agua y la concentración de humedad (Twidale, 1982; Campbell, 1997). Durante otras fases de la morfogénesis, algunos investigadores conceden un papel dominante a la crioclastia (Ikeda, 1990); otros defienden la importancia de la cristalización de sales, procedentes de la propia roca (Bradley et al., 1978) o de fuentes externas (Leyton y Andrade, 1987); en los medios áridos, consideran el factor de los recubrimientos en la superficie externa (óxidos de hierro, manganeso) para explicar los estados actuales de las formas.

El estudio de la interacción de los procesos que modifican el estado de la forma en el tiempo, contribuye al conocimiento de su desarrollo polifásico (regulado por una serie de variables). La interpretación del origen mantiene hipótesis múltiples. La aplicación de técni-

Figura 3

TIPOLOGÍA DINÁMICA

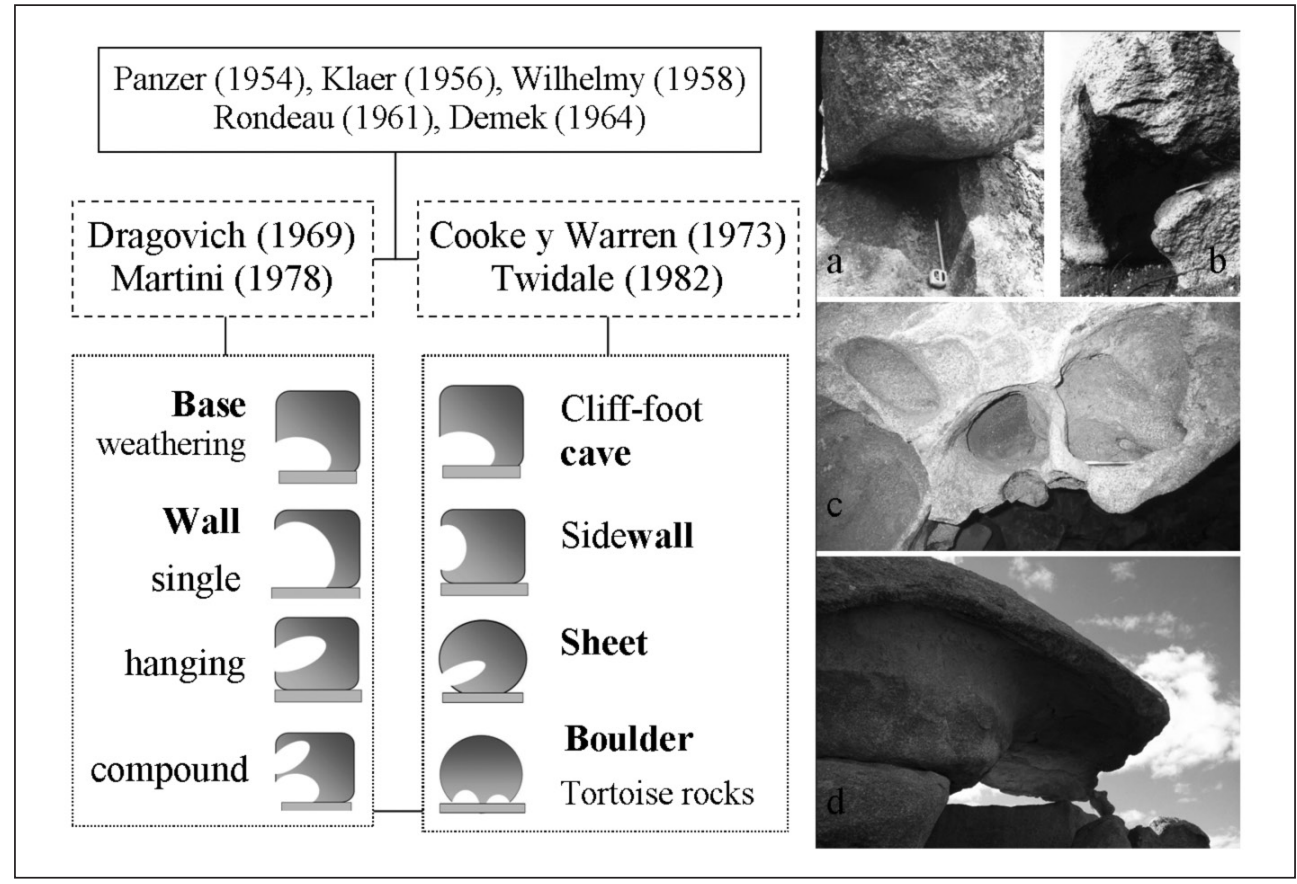

Tipos en Galicia: a) Castro Santomé, Ourense b) Monte Fieiteira, A Coruña c) A Merca, Ourense; foto d) Los Riojanos, noroeste de Sierras Pampeanas, Argentina (altura de la cavidad 1,4 metros). 
cas estadísticas favorece la identificación de los parámetros significativos en ambos casos. Sancho \& Benito (1990 o.c.), en las areniscas de la cuenca del Ebro, con los registros de dimensión de las cavidades (longitud, anchura, profundidad) y de los factores en su morfogénesis (propiedades de la roca, orientación, altura, inclinación de la superficie) categorizan los estados presentes (agrupamientos). Del análisis de correlación y regresión múltiple de todos los valores obtienen dos combinaciones significativas: la composición de la roca con la inclinación de la superficie para explicar la densidad de las formas; la fábrica rocosa con la red de discontinuidades para explicar el volumen erosionado mediante procesos de haloclastia y humectación-desecación. Los estadísticos, indicadores del cambio en el crecimiento, permiten detectar fenómenos de perpetuación, diversificación o destrucción de un estado previo. Sin embargo, la selección de los estimadores (clásicos o resistentes) implica la comprobación del ajuste de los datos a un tipo de distribución. Avanzar en el conocimiento de la diversidad requiere además examinar sus modalidades de comportamiento.

\subsection{Dinámica no lineal}

La demostración de que el comportamiento de estas formas tiende a la irregularidad tiene sus antecedentes en los ensayos de laboratorio de Colman (1981) sobre la relación meteorización-tiempo: resultan funciones logarítmicas, salvo para el supuesto de la disolución de rocas calizas (de tendencia lineal). Los primeros datos de profundidad de los tafoni utilizados para comprobarlo proceden de los registros en tres niveles de plataforma costera (areniscas y conglomerados), emergidos por actividad sísmica en Boso (Japón); el más antiguo, con una edad de exposición subaérea entre 1.300-1.500 años. Los datos se ajustaban a la ecuación exponencial (Matsukura y Matsuoka, 1991: 55)

$$
D=20,3\left(1-\mathrm{e}^{-0,005 t}\right)
$$

donde $D$ es la media de los 10 casos más profundos (cm) y $t$ es el tiempo (años), con una tasa máxima de 1,67 $\mathrm{mm} \mathrm{a}^{-1}$ para un tiempo de exposición de 1.400 años; la relación es lineal entre $D$ y $t$ para los primeros 66 años. Mottershead y Pye (1994) con la misma medida en las cavidades de la costa de Devon (Inglaterra) confirmaban un comportamiento similar en los esquistos.

Sunamura (1996) argumenta que, tras la exposición de un volumen rocoso en superficie, existe un tiempo variable en el que se reduce su resistencia inicial a la meteorización ( $\mathrm{t}^{*}$ ); alcanzado un coeficiente menor que el inicial ( $S^{*}$ en un tiempo $t>t^{*}$ ) es posible la generación de las formas. La tasa máxima de crecimiento corresponde al inicio $\left(t=t^{*}\right)$. El cambio de la profundidad $(z)$ en medios costeros, siendo $z_{\mathrm{c}}$ la profundidad máxima de los casos, estaría descrito por la ecuación (Sunamura, 1996 o.c.: 745)

$$
z=z_{\mathrm{c}}\left(1-\left(t^{*} / t\right)^{0,5}\right)
$$

El control ejercido por la resistencia de la roca frente a diversos esfuerzos ya había sido analizado en sólidos cristalinos. Vidal Romaní $(1985,1989)$ propone un modelo de formación de cavidades a partir de coeficientes críticos de dislocación, bajo cargas concentradas, 
en ambiente endógeno: la generación de recintos de colapso, con movimientos diferenciales a escala granular, delimita áreas más sensibles a la meteorización y la erosión; dirigen la configuración de los tafoni una vez que la roca aflora en superficie.

\section{Significado de la complejidad}

La distribución espacial de los tafoni, en un volumen rocoso dado, delimita sectores donde la erosión sobrepasa la tasa esperada en función de la litología y del clima. La erosión no afecta por igual a todos los elementos de la forma durante su desarrollo: es más intensa en las paredes internas y en la bóveda que en la superficie externa. La dinámica del sistema genera una complejidad creciente por las modificaciones que experimentan sus componentes y las relaciones con el entorno; la complejidad define un tejido de orden y desorden (Ibáñez et al., 1995; Morin, 1998). En este marco, los debates relacionados con la naturaleza de los procesos pasan a segundo plano (Malanson, 1999; Murray et al., 2009) ya que las claves de la interpretación de un sistema son las conexiones: entre las condiciones de origen, la dimensión y la morfología. Se revisan las teorías que han sustentado la explicación de estas formas en dos etapas (Twidale, 2002), o los conceptos que las definen como pseudo-karst en rocas no calizas (Wray, 1997; Self y Mullan, 2005) por la importancia relativa de los procesos de disolución.

\subsection{Comportamiento}

Los principios de termodinámica para sistemas abiertos alejados del equilibrio (Nicolis \& Prigogine, 1977) fundamentan la definición del comportamiento complejo. La mayoría de estos sistemas poseen un límite natural de crecimiento en el tiempo. Su evolución mediante fluctuaciones genera estados de orden y desorden, con funciones acopladas complejas. El comportamiento del sistema depende de las condiciones de sus límites hasta que alcanza ciertas dimensiones (Prigogine, 1983; Huggett, 1988); entonces, los cambios generados por la dinámica interna son los más importantes y los efectos de perturbaciones locales pueden ser amplificados. La interpretación de estos sistemas prescinde de las barreras entre los elementos bióticos y abióticos (Jacob, 1988). La red de interacciones que los sustenta los conecta a través de diversas escalas (Culling, 1987; Viles, 1988; Holling, 1992) en jerarquías dinámicas anidadas (Werner, 1999). Las transformaciones morfológicas de los tafoni describen trayectorias complejas con diversas modalidades de comportamiento entre su génesis y su colapso funcional.

Mellor et al. (1997) las resumen en cuatro etapas para los tipos laterales (en areniscas): iniciación, a partir de las irregularidades del volumen rocoso; crecimiento, por desagregación granular o descamación combinadas con deflación eólica (gobernado por fenómenos de retroalimentación positiva); amalgamación, con procesos de coalescencia que borran las huellas de la etapa anterior en la superficie interna de la bóveda; y degradación, que expone la bóveda a los agentes ambientales. Twidale y Vidal (2005) consideran igualmente cuatro etapas para los tipos basales (en granitos): iniciación, entre dos planos de una discontinuidad; crecimiento rápido en altura, con diversificación de la superficie interna de la bóveda (protuberancias, alveolos); ralentización del crecimiento, con coalescencia de alveolos en la 
bóveda y posible apertura de un orificio al exterior; y fragmentación de la superficie externa, con la configuración de una visera o alero que cierra parcialmente la bóveda expuesta.

La complejidad emerge en la red de interacciones tectónica-meteorización-erosión. Integra fases convergentes y divergentes, conectadas con la dinámica de otros componentes del relieve (residuales, morrenas, plataformas, escarpes, laderas); una situación difícilmente reducible a pocos parámetros. La auto-replicación de la morfología inicial (McBride y Picard, 2000) se detiene cuando la forma alcanza una configuración determinada, en la que sus componentes evolucionan con magnitudes diferenciales, y comienza la tendencia hacia la diversificación. El crecimiento de en profundidad en condiciones litológicas y climáticas homogéneas es más rápido en los tipos laterales (De Uña Álvarez, 2004). Por lo tanto las categorías morfológicas son significativas en el contexto del estudio de sus trayectorias dinámicas, analizadas en diferentes escenarios.

\subsection{Trayectorias}

Las trayectorias reflejan el grado de acoplamiento estructural y funcional entre los componentes de la forma y el entorno (Lageat \& Gunnell, 2001; Brunsden, 2001; Wainwright et al., 2011). En este sentido los tafoni son sistemas naturales con inestabilidad dinámica, hasta que alcanzan las mayores dimensiones en el espacio disponible (Phillips, 2003; Viles, 2005) independientemente de rocas o procesos específicos. Norwick y Dexter (2002) demuestran, en las calizas y areniscas de Colorado (EE.UU.), que los datos de su variabilidad morfológica - para unidades rocosas con la misma edad de exposición, topografía y clima - se ajustan a una curva sigmoidal expresada por la ecuación (Norwick y Dexter, 2002 o.c.: 22)

$$
D=b 1+\mathrm{e}^{(b 2+(b 3 / t))}
$$

donde $D$ es la profundidad media de los tafoni de mayor dimensión en una muestra, $t$ es la edad de las unidades que los hospedan y $b 1, b 2, b 3$ son constantes a resolver para diversos tiempos de retardo $t^{*}$ (máximo 49.000 años) y coeficientes $S^{*}$ variables en función de las facies litológicas. La tasa decrece a lo largo de decenas de miles de años, aunque cabe la divergencia de esta tendencia general con retroalimentación positiva en sus inicios y retroalimentación negativa en su límite.

La reformulación para obtener la tasa de cambio de la profundidad en el tiempo $(Z)$, mediante el ajuste a una curva sigmoidal (Sunamura y Aoki, 2011: 1), remite a la ecuación

$$
Z=Z_{c}[1-(n+1) \exp (-\beta t)+n \exp (-(1+1 / n) \beta t)]
$$

donde $Z_{\mathrm{c}}$ es la profundidad máxima alcanzada por las formas, $t$ el tiempo, $\beta$ y $n$ constantes (relativas a las variables de control del crecimiento); estos autores utilizan datos de siete estaciones de muestreo, incluyendo los de Norwick y Dexter (2002 o.c.), localizadas desde medios costeros hacia el interior desértico, con procesos dominantes de haloclastia. Una secuencia de especial interés está delimitada por $t=t^{* *}=0,9 Z_{\mathrm{c}}$ resultando un tiempo aproximado de $10^{5}$ años para que un tafone alcance el $90 \%$ de su profundidad límite en ambiente desértico. La tasa máxima corresponde al inicio del crecimiento, encontrándose entre el 25\% 
del tiempo requerido para su desarrollo casi completo. Puede variar en función de los coeficientes de resistencia de la roca y de las condiciones ambientales.

Las trayectorias de los tafoni presentan las mismas propiedades (fases de comportamiento lineal y no lineal, rápidas y lentas) en litologías, regímenes geodinámicos y ambientes muy diferentes (Fig. 4). La distribución de los datos de profundidad (P en $\mathrm{cm}$ ) en muestras de los afloramientos graníticos en el noroeste de la Península Ibérica, o la del volumen rocoso erosionado $\left(\mathrm{V}\right.$ en $\mathrm{m}^{3}$ ) en muestras de los granitos de las Sierras Pampeanas en el noroeste argentino, confirman una tendencia similar. Los cambios están relacionados con las dimensiones alcanzadas y las modificaciones de la morfología durante el crecimiento. Es decir, la diversificación está limitada por diseños funcionalmente activos. Existen etapas en las que algunos componentes se transforman con mayor rapidez, como sucede con la bóveda en las primeras fases; y etapas en las que se modifica su papel en el cambio, como sucede cuando la bóveda es expuesta al aire libre en las últimas fases y los agentes subaéreos controlan su degradación.

TENDENCIAS EVOLUTIVAS

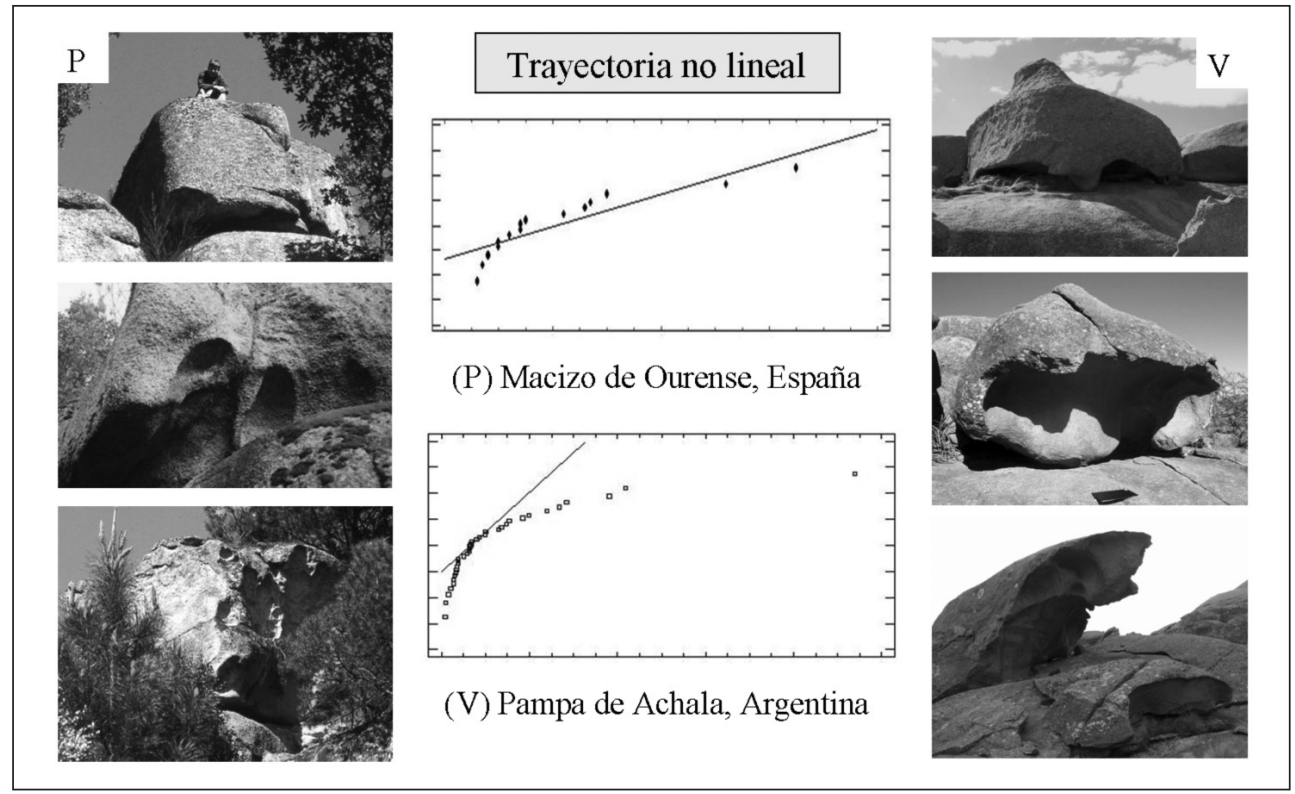

Profundidad (P) en formas incipientes del replano de Sabadelle (Ourense, Galicia) con valor máximo de 0,6 m (foto inferior izquierda). Volumen de roca erosionada (V) en formas evolucionadas de la Pampa de Achala (Argentina) con P máxima de 3,9 m (foto inferior derecha).

Los tafoni articulan una red de nodos de la erosión en un amplio rango de escalas espacio-temporales. French y Gugliemin (2002) afirman que los únicos procesos verdaderamente activos en las superficies de cumbre del Monte Keinath (Antártida) son los relacionados con su evolución (gobernada por los efectos del estrés térmico en los cristales de la roca); las 
cavidades crecen bajo un barniz de óxidos de hierro, procedentes de las biotitas, y cuando lo rompen capturan el fondo de formas tipo gnamma desarrolladas sobre él. Hall y André (2006) demuestran que los efectos del estrés térmico en la roca presentan magnitudes diferenciales a escala granular o de superficie. Strini et al. (2008) aportan datos, en el mismo lugar, sobre la intensidad relativa de este fenómeno (menor en los bloques erráticos) concluyendo que su dinámica responde sobre todo a fluctuaciones de corto plazo en el estrés térmico y la acción de la haloclastia en la cavidad interna (una escala donde la crioclastia no es activa y la mayor o menor velocidad del viento determina la humedad). Brandmeier et al. (2011), apoyándose en dataciones de ${ }^{10} \mathrm{Be}$, obtienen en Córcega tasas de meteorización - para las formas más antiguas - con un ratio entre 3 y 25 veces superior a las de las superficies adyacentes; el análisis de la temperatura, humedad relativa, dimensiones y colonias de líquenes en su interior muestra la detención del proceso en diferentes momentos para diversos sectores de la cavidad (sin relación con la altitud o la distancia al mar).

El impacto de los procesos varía a lo largo de la trayectoria de la forma, relacionado con las condiciones de la unidad en donde se localiza. En los tor de Corea, Matsukura y Tanaka (2000) distinguen los bloques con probabilidad de generar cavidades por el menor coeficiente de resistencia de la roca y el mayor contenido en humedad; en su base, la meteorización química y la evaporación periódica del agua (capilar o de lluvia) potencia los efectos de la haloclastia, originando las formas; delimitadas de nuevo por superficies internas de menor resistencia y mayor humedad que la exterior, las etapas del crecimiento reflejan la influencia de la variación de esos parámetros en diferentes sectores de la cavidad. En los tor del noroeste de la Península Ibérica, las dimensiones de los bloques y los parámetros de la carga ejercida por su peso están directamente relacionados con la configuración morfológica de las cavidades.

La importancia de la fábrica y del sistema de discontinuidades de la roca en la génesis, crecimiento y degradación de los tafoni se reconoce en los trabajos que analizan el fenómeno en diversas escalas (Baonza Díaz, 1999; McBride y Picard, 2000 o.c.; Turkington y Phillips, 2004; De Uña Álvarez y Vidal Romaní, 2008; Dill et al., 2010). La intensidad de los efectos en la meteorización y la erosión del relieve a largo plazo está regulada por sus transformaciones dimensionales, morfológicas o funcionales. El comportamiento como sistemas naturales complejos hace que presenten con frecuencia estados de transición entre configuraciones estables e inestables (Galán, 2010; Phillips, 2011). Una cavidad que crece en el interior de un bloque rocoso de posición estable sobre una superficie puede modificar los esfuerzos que lo mantienen como elemento residual; una cavidad que crece en un bloque de posición estable en una ladera, puede modificar o detener su desarrollo al variar las condiciones de su ángulo de reposo.

De la dinámica de la morfogénesis resultan una serie de patrones morfológicos, indicativos de la organización de interacciones complejas. Considerando las interacciones abióticas/ bióticas con sus parámetros de control, la morfología muestra proporciones de cambio isométricas (auto-semejanza litológica y climática) y alométricas (auto-afinidad en la distorsión de la forma) en diversas escalas. El carácter azonal y las trayectorias evolutivas, con propiedades de auto-organización, hacen difícil definir un origen solo mediante la combinación de procesos de meteorización y de erosión. Desde un planteamiento holístico, la interpretación de su génesis tiene en cuenta los posibles campos generativos en una masa rocosa. 
Un campo generativo identifica el morfo-espacio, las condiciones iniciales, determinando tanto los límites de tamaño y morfología como su grado de exposición a un conjunto de procesos (endógenos, epigénicos, subaéreos). La interpretación de su desarrollo centra la investigación en la evolución de los patrones morfológicos, conectada con la de otros componentes del relieve. Los patrones invariantes representan secuencias de cambio unidireccional; son característicos de la morfogénesis robusta (auto-replicada) en los sistemas no lineales: mantienen una configuración morfologica que conserva las relaciones morfométricas durante su evolución (aunque presenten diferentes dimensiones). Los patrones variantes representan secuencias de cambio multidireccional; caracterizan en la morfogénesis las bifurcaciones del sistema: la configuración morfológica experimenta transformaciones que alteran las relaciones morfométricas durante su evolución. La relación de ambos en una trayectoria se expresa por los parámetros matemáticos de tendencia (ajuste a una distribución), curvatura (magnitud derivada) y suavidad (grado de oscilación). La segmentación de una trayectoria en patrones diferenciados se expresa por las medidas estadísticas resistentes (rango, mediana, valores límite).

\section{CONCLUSIÓN}

La interpretación de los tafoni ha generado un conjunto de categorías nominales, definidas por rasgos dimensionales y morfológicos. La diferenciación de tipos por el criterio de analogía parte de los registros cualitativos y cuantitativos, comparados en diferentes escenarios. La explicación está relacionada con el concepto de la forma como un sistema proceso-respuesta, bajo control de las propiedades de las rocas y de condiciones ambientales específicas. A lo largo del tiempo, la incertidumbre sobre un origen común y el conocimiento sobre los procesos de su desarrollo revelan la dificultad del análisis con parámetros simples. La interpretación basada en múltiples variables y sucesos interdependientes asume el concepto de la forma como un sistema dinámico, de comportamiento no lineal. El análisis de los datos cualitativos y cuantitativos, en el contexto de diferentes unidades del relieve, demuestra su naturaleza compleja.

El estudio de la diversidad posibilita la detección de condiciones críticas en la permanencia y el cambio: la micro-macro dislocación del material de partida, el potencial de transformación frente a las fluctuaciones internas o externas. Conceptos previos, como el de equilibrio o el de estabilidad en sistemas naturales abiertos, se revisan en el contexto de la evolución del relieve a largo plazo. La interpretación se fundamenta en la teoría de los sistemas complejos; define los tafoni en términos de inestabilidad condicionada, con diverso grado de acoplamiento estructural y funcional en otras unidades del relieve. Las categorías morfológicas representan estados momentáneos del sistema. El análisis de las trayectorias evolutivas favorece el conocimiento de su desarrollo polifásico. El significado de las categorías está relacionado con las modalidades de su comportamiento.

La distribución espacial de estas formas delimita volúmenes rocosos diferenciados por la sensibilidad frente a la meteorización y la erosión. Actúan como nodos de propagación de estos procesos de manera puntual (en la propia forma), lineal (canalizada por las discontinuidades de la roca) y difusa (a través de superficies o laderas). Su interpretación requiere un enfoque holístico. Considera el comportamiento de las unidades que hospedan las formas 
(bloques, paredes inclinadas) y de los componentes de su configuración (fondo, paredes, bóveda, superficie externa).

Las variaciones en la composición o estructura de la roca, la interacción de una serie de procesos, la localización en altitud o la posición más o menos cercana al mar no explican por sí solas el origen de estas formas menores. Estas variables intervienen en la morfogénesis con diferentes magnitudes en función de la escala espacio-temporal. Así lo demuestran las ecuaciones de crecimiento propuestas en condiciones litológicas, climáticas y topográficas contrastadas. La existencia de varios niveles de auto-organización se refleja en los últimos trabajos que tratan el tema. Orienta la investigación hacia las secuencias de cambio, conectadas con los posibles campos generativos, y las trayectorias que generan patrones morfológicos complejos. La interpretación de los tafoni plantea todavía muchas preguntas sobre su génesis y su dinámica de cambio en diferentes escalas.

\section{BIBLIOGRAFÍA}

BAONZA DÍAZ, J. (1999): «Varios tafoni singulares en la Sierra de Guadarrama Bustarviejo, Madrid)». Cuaternario y Geomorfología, 13(1-2), 53-62.

BLACKWELDER, E. (1929): «Cavernous Rock Surfaces of the Desert». American Journal of Science, 101, 393-399.

BIROT, P. (1958): «Les dômes cristallines». Mémoires et Documents du C.N.R.S., tome VI, 9-34.

BOURCART, J. (1930): «Le problème des taffoni de Corse et l'érosion alvéolaire». Revue de Géographie Physique et de Géologie Dynamique, III(1), 5-15.

BRADLEY, W.C., HUTTON, J.T. y TWIDALE, C.R. (1978): «Role of salts in development of granitic tafoni, south Australia». Journal of Geology, 86, 647-654.

BRANDMEIER, M., KUHLEMANN, J., KRUMREI, I., KAPPLER, A. Y KUBIK, P.W. (2011): «New challenges for tafoni research: A new approach to understand processes and weathering rates». Earth Surface Processes and Landforms, 36(6), 839-852.

BRUNSDEN, D. (1996): «Geomorphological Events and Landform Change». Zeitschrift für Geomorphologie, 40(3), 273-288.

BRUNSDEN, D. (2001): «A critical assessment of the sensitivity concept in geomorphology». Catena, 42, 99-123.

BRUNSDEN, D. y THORNES, J.B. (1979): «Landscape sensitivity and change». Transactions of the Institute of British Geographers, 4, 463-484.

BRYAN, K. (1928): «Niches and other cavities in sandstone at Chaco Canyon». Zeitschrift für Geomorphologie, 3, 125-140.

CAILlEUX, A. (1953): «Taffonis et érosion alvéolaire». Cahiers Géologiques de Thoiry, $16 / 17,130-133$.

CALKING, P. y CAILLEUX, A. (1962): «A quantitative study of cavernous weathering (taffonis) and its application to glacial chronology in Victoria Valley, Antarctica». Zeitschrift für Geomorphologie, 6, 317-324.

CAMPBELL, E.M. (1997): «Granite Landforms». Journal of the Royal Society of Western Australia, 80, 101-112. 
CAYEUX, L. (1911): «Description physique de l'Ille de Délos». Exploration archéologique de Délos - École française d'Athènes, Paris (I) 4, 162-186.

COLMAN, S.M. (1981): «Rock-Weathering Rates as Functions of Time». Quaternary Research, 15, 250-264.

COOKE, R. y WARREN, A. (1973): Geomorphology in deserts. The University California Press. 374 pp.

CULLING, W.E.H. (1987): «Equifinality: Modern Approaches to Dynamical Systems and their Potential for Geographical Thought». Transactions of the Institute of British Geographers NS, 12(1), 57-72.

CHOFFAT, P. (1895): «Notes sur l'érosion en Portugal: Sur quelques cas d'érosion atmospherique dans les granites du Minho (Tafoni)». Com. Servicios Geológicos de Portugal, 3, 17-26.

CHOLLEY, A. (1950): «Morphologie structurale et morphologie climatique». Annales de Géographie, 59(317), 321-335.

CHORLEY, R.J. (1962): Geomorphology and General Systems Theory. United States Geological Survey. Professional Paper. 500-B.

CHORLEY, R.J., DUNN, A.J. y BECKINSALE, R.P. (1964): The History of the Study of Landforms. London. Methuen. $678 \mathrm{pp}$.

CHORLEY, R.J. y KENNEDY, B.A. (1971): Physical Geography: A systems approach. London. Prentice Hall. 370 pp.

CHURCH, M. (2010): «The trajectory of geomorphology». Progress in Physical Geography, 34(3), 265-286.

DEMEK, J. (1964): «Slope development in granite areas of Bohemian massif (Czechoslovakia) ». Zeitschrift für Geomorphologie, 8, 82-106.

DENAEYER, M.E. (1953): «Erosion alvéolaire et taffoni du Cap de Creus (Province de Gerona, Pyrennées orientales catalanes) et autres lieux». Bulletin de la Societé Belge de Géologie, 62 (2/3), 194-210.

DRAGOVICH, D. (1969): «The origin of cavernous surfaces (tafoni) in granitic rocks of southern South Australia». Zeitschrift für Geomorphologie, 13, 163-181.

DE UÑA ÁLVAREZ, E. (2004): «Tafoni en rocas graníticas. Primera valoración estadística sobre las tasas de desarrollo en el macizo de Ourense (Galicia, NW de la Península Ibérica)». Cadernos do Laboratorio Xeolóxico de Laxe, 29, 265-289.

DE UÑA ÁLVAREZ, E. y VIDAL ROMANÍ, J.R. (2008): «Some minor features (tafoni, cavernous forms) in the granite terrains of Los Riojanos (Pampa de Achala, Sierra Grande de Córdoba, República Argentina). Geometric and morphologic properties». Cadernos do Laboratorio Xeolóxico de Laxe, 33, 83-99.

EVANS, I.S. (1970): «Salt crystallization and rock weathering: a review». Revue de Géomorphologie Dynamique, 19(4), 153-176.

DILL, H.G., WEBER, B. y GERDES, A. (2010): «Constraining the physical-chemical conditions of Pleistocene cavernous weathering in Late Paleozoic granites». Geomorphology, 121, 283-290.

EDESO, J.M. (1988): «Microformas en las areniscas eocenas de la formación Jaizkibel». Lurralde, 11, 57-84. 
FRENCH, H.M. y GUGLIELMIN, M. (2002): «Observations on Granite Weathering Phenomena, Mount Keinath, Northern Victoria Land, Antarctica». Permafrost and Periglacial Processes, 13, 231-236.

FUTTERER, K. (1902): «Der Per-schan als Typus der Felsenwüste». Geographische Zeitschrift, 8, 323-339.

GALÁN, C. (2010): «Patrones y estructuras disipativas en cuevas y geoformas del pseudokarst de Jaizkibel». Sociedad de Ciencias Aranzadi. Disponible en http://www.aranzadi-zientziak.org/fileadmin/docs/espeleologia/EstrucDisip.2.TOTAL.pdf.

GERRARD, A.J. (1984): «Multiple working hypotheses and equifinality in Geomorphology: Comments on the recent article by Haines-Young and Petch». Transactions of the Institute of British Geographers NS, 9(3), 364-366.

GOUDIE, A.S. (2004): «Tafoni» en Encyclopedia of Geomorphology (Goudie, A.S., ed.). London, Routledge, vol. 2, 1034-1035.

GODARD, P. (1977): Pays et paysages du granite. Paris. P.U.F. 232 pp.

GREGORY, K.J. (1985): The nature of Physical Geography. London. Arnold. 262 pp.

GREGORY, K.J. y GOUDIE, A.S. (2011): The Handbook of Geomorphology. London. Sage. $610 \mathrm{pp}$.

GRENIER, M.P. (1968): «Observations sur les taffonis du désert chilien». Bulletin de l'Association des Géographes Français, 364/365, 193-211.

GUTIÉRREZ ELORZA, M. (2008): Geomorfología. Pearson. Prentice-Hall, 920 págs.

GUTIÉRREZ, M. y PEÑA, J.L. (1988): Perspectivas en Geomorfología. Zaragoza. Monografías de la Sociedad Española de Geomorfología. 223 pp.

HALL, K. y ANDRÉ, M.F. (2006): «Temperature observations in Antartic tafoni: implications for weathering, biological colonization and tafoni formation». Antartic Science, 18(3), 377-384.

HAINES-YOUNG, R.H. y PETCH, J.R. (1983): «Multiple work hypotheses: Equifinality and the study of landforms». Transactions of the Institute of British Geographers NS, 8(4), 458-466.

HAINES-YOUNG, R.H. y PETCH, J.R. (1986): Physical Geography: Its nature and methods. London. Harper \& Row. 230 pp.

HÖLLERMANN, P. (1975): «Formen kavernöser verwitterung (tafoni) auf Teneriffa». Catena, 2, 385-410.

HOLLING, C.S. (1992): «Cross-scale Morphology, Geometry, and Dynamics of Ecosystems». Ecological Monographs, 62(4), 447-502.

HUGGETT, R.J. (1988): «Dissipative systems: implications for Geomorphology». Earth Surface Processes and Landforms, 13, 45-49.

HULT, R. (1899): «Granitens vittring i Galicien». Meddelanden af Geografiska Föreningen i Finland, V, 1-17.

HUME, W.F. (1925): The surface features of Egypt, their determining causes and relation to geological structure. Government Press. 408 pp.

IBÁÑEZ, J.J., DE ALBA, S. y GARCÍA ÁLVAREZ, A. (1995): «Aportaciones del caos a las Ciencias de la Tierra (estructura, evolución y dinámica del modelado terrestre)» en Análisis de la variabilidad espacio-temporal y procesos caóticos en ciencias medioambientales (Ibáñez, J.J. y Machado, C., eds.). Logroño, Geoforma ediciones, 43-80. 
IKEDA, H. (1990): «Tafoni topography and its development process as seen in the Jhumonjin area of the north-eastern coastal Korean Peninsula». Memoirs of Nara University, 18, 49-66.

INKPEN, R. (2005): Science, Philosophy and Physical Geography. London. Routledge. 164 pp.

JACOB, F. (1988): La lógica de lo viviente. Barcelona. Biblioteca Científica Salvat, 337 pp.

JUTSON, J.T. (1917): «Erosion and the resulting land forms in Sub-Arid Western Australia». The Geographical Journal, 50(6), 418-434.

KEJONEN, A., KIELOSTO, S. y LAHTI, S.I. (1988): «Cavernous weathering forms in Finland». Geografiska Annaler, 70A (4), 315-322.

KELLETAT, D. (1980): «Studies on the Age of Honeycombs and Tafoni features». Catena, 7, 317-325.

KENNEDY, B.A. (2006): Inventing the Earth: ideas on landscape development since 1740. Chichester. Wiley-Blackwell. 160 pp.

KLAER, W. (1956): Verwitterungsformen im granit auf Korsika. Pettermans Geografische Mitteilungen, 261, 1-146.

KVELBERG, I. y POPOFF, B. (1938): «Die Tafoni Verwitterungsercheinung». Latvijas Univ. Raksti, 6, 129-370.

LAGEAT, Y. y GUNNELL, Y. (2001): «Structural Predesign and Scaling Factors in Geomorphology» en Basement Regions (Godard, A.; Lagasquie, J.J. y Lageat, Y., eds.). Berlín, Springer-Verlag, 259-272.

LEYTON LÓPEZ, L. y ANDRADE JOHNSON, B. (1987): «Morfología de Cavidades en la Costa de Chile Central». Revista de Geografía Norte Grande, 14, 21-34.

LÓPEZ BERMÚDEZ, F. (2002): «Geografía física y conservación de la naturaleza». Papeles de Geografía, 36, 133-146.

MAINGUET, M. (1972): Le modelé des grès. Paris. Institut Géographique National, 657 págs.

MARTÍNEZ DE PISÓN, E. (1975): «Reflexión sobre el realismo geomorfológico». Estudios Geográficos, 36(140/141), 697-741.

MARTINI, I.P. (1978): «Tafoni weathering with examples from Tuscany, Italy». Zeitschrift für Geomorphologie, 22(1), 44-67.

MATSUKURA, Y. y MATSUOKA, N. (1991): «Rates of tafoni weathering on uplifted shore platforms in Nojima-Zaki, Boso Peninsula, Japan». Earth Surface Processes and Landforms, 16, 51-56.

MATSUKURA, Y. y TANAKA, Y. (2000): «Effect of rock hardness and moisture content on tafoni weathering in the granite of Mount Doeg-Sung, Korea». Geografiska Annaler, 82A, 59-67.

McBRIDE, E.F. y PICARD, M.D. (2000): «Origin and development of tafoni in Tunnel Spring Tuff, Crystal Peak, Utah, USA». Earth Surface Processes and Landforms, 125, 869-879.

MELLOR, A., SHORT, J. y KIRKBY, S.J. (1977): «Tafoni in the El Chorro area, Andalucía, southern Spain». Earth Surface Processes and Landforms, 22, 817-833.

MORIN, E. (1998): Introducción al pensamiento complejo. Barcelona. Gedisa. 168 pp. 
MOTTERSHEAD, D.N. y PYE, K. (1994): «Tafoni on Coastal Slopes, South Devon, UK». Earth Surface Processes and Landforms, 19, 543-563.

MUÑOZ JIMÉNEZ, J. (1992): Geomorfología general. Madrid. Síntesis. 351 pp.

MURRAY, B., LAZARUS, E., ASHTON, A., BAAS, A., COCO, G., COULTHARD, T., FONSTAD, M., HAFF, P., McNAMARA, D., PAOLA, C., PELLETIER, J. y REINHARDT, L. (2009): «Geomorphology, complexity, and the emerging science of the Earth's surface». Geomorphology, 103, 496-505.

MUSTOE, G.E. (1983): «Cavernous Weathering in the Capitol Reef desert, Utah». Earth Surface Processes and Landforms, 8, 517-526.

NICOLIS, G. y PRIGOGINE, L. (1977): Self-organization in nonequilibrium systems: from dissipative structures to order through fluctuations. New York. Wiley. 491 pp.

NICHOLS, R.L. (1953): Geomorphology of Marguerite Bay, Palmer Peninsula, Antarctica. Washington: Office Naval Research Technical Reporter (12).

NORWICK, S.A. y DEXTER, L.R. (2002): «Rates of development of tafoni in the Moenkopi and Kaibab formations in Meteor crater and on the Colorado plateau, Northeastern Arizona». Earth Surface Processes and Landforms, 27, 11-26.

OTTMANN, F. (1956): «Sur l’âge de quelques taffoni en Corse». Bulletin de la Société Géologique de France, 6, 62-64.

PANZER, W. (1954): «Verwitterungs und abtragungsformen im granit von Hong Kong». Mortesen-Festschrift, Veröff. Akad., 41-60.

PASSARGE, S. (1920): Die Grundlagen der Landschaftskunde. Hamburg. Friederischsen, $558 \mathrm{pp}$.

PENCK, A. (1894): Morphologie der Erdoberfläche. Stuttgart. Engelhorns. Vol. I, 214 pp.

PHILLIPS, J.D. (2003): «Sources of nonlinearity and complexity in geomorphic systems». Progress in Physical Geography, 27(1), 1-23.

PHILLIPS, J.D. (2011): «Emergence and pseudo-equilibrium in geomorphology». Accepted Manuscript, Geomorphology, doi: 10.1016/j.geomorph.2011.05.017

PRADO, C. de (1976): Descripción física y geológica de la provincia de Madrid (1864). Madrid. Edición del Colegio de Ingenieros, Canales y Puertos. Colección Ciencias, Humanidades e Ingeniería, 2, 352 pp.

PREBBLE, M.M. (1967): «Cavernous weathering in the Taylor Dry valley, Victoria Land, Antarctica». Nature, 216, 194-195.

PRIGOGINE, I. (1983): ¿Tan solo una ilusión? Una exploración del caos al orden. Barcelona. Tusquets. 325 pp.

REYNAUD, A. (1971): Epistémologie de la Géomorphologie. Paris. Masson. 126 pp.

REUSCH, H. (1882): «Notes sur la géologie de la Corse». Bulletin de la Société Géologique de France, XI, 53-67.

RHOADS, B.L. y THORN, C.E. (1996): The Scientific Nature of Geomorphology. Chichester. Wiley \& Sons. 481 pp.

RODRÍGUEZ VIDAL, J. y NAVASCUÉS, L. de (1982): «La tafonización de las areniscas miocenas en los alrededores de Huesca». Tecniterrae, 19, 7-12.

RÖGNER, K. (1988): «Measurements of Cavernous Weathering at Machtesh Hagadol (Negev, Israel). A semiquantitative study». Catena, 12, 67-76. 
RONDEAU, A. (1961): Recherches géomorphologiques en Corse. Paris. Armand Colin. 586 pp.

SANCHO, C. y BENITO, G. (1990): «Factors controlling Tafoni weathering in the Ebro Basin (NE Spain) ». Zeitschrift für Geomorphologie, 34 (2), 165-177.

SCHEIDEGGER, A.E. (1961): Theoretical Geomorphology. Berlin. Springer-Verlag. 334 pp.

SCHUMM, S.A. y LICHTY, R.W. (1965): «Time, space and causality in geomorphology». American Journal of Science, 263, 110-119.

SANZ HERRAINZ, C. (1976): «La morfología de la Pedriza de Manzanares». Estudios Geográficos, 145, 435-463.

SEGERSTROM, K. y HENRIQUEZ, H. (1964): «Cavities or tafoni in rock faces of the Atacama desert, Chile». United States Geological Survey, 501-C, 121-125.

SELF, C.A. y MULLAN, G.J. (2005): «Redefining the boundary between Karst and Pseudokarst». Cave and Karst Science, 23 (2), 63-70.

SMITH, B.J. (1978): «The origin and geomorphic implications of cliff foot recesses and tafoni on limestone hamadas in the northwest Sahara». Zeitschrift für Geomorphologie, 22 (1), 21-43.

SORIANO, M.A. (1987): «Características de los tafonis y alveolos desarrollados al sur de Zaragoza». Revista de la Academia de Ciencias de Zaragoza, 42, 123-131.

SPEDDING, N. (1997): «On growth and form in Geomorphology». Earth Surface Processes and Landforms, 22, 261-265.

STRINI, A., GUGLIELMIN, M. y HALL, K. (2008): «Tafoni development in a cryotic environment: an example from northern Victoria land, Antarctica». Earth Surface Processes and Landforms, 33, 1502-1519.

SUMMERFIELD, M.A. (2005): «A tale of two scales, or the two geomorphologies». Transactions of the Institute of British Geographers, 30, 402-415.

SUNAMURA, T. (1996): «A Physical Model for the Rate of Coastal Tafoni Development». The Journal of Geology, 104, 741-748.

SUNAMURA, T. y AOKI, H. (2011): «Application of an S-shaped curve model to the temporal development of tafoni of salt-weathering origin». Accepted Article, Earth Surface Processes and Landforms, doi: 10.1002/esp.2175.

TEODOROPOULOS, D. (1974): «Tafoni weathering forms of Tinos Island». Annales Géologiques des Pays Helleniques, 26, 149-158.

THORN, C.E. (1988): An Introduction to Theoretical Geomorphology. Boston. Unwin. 247 pp.

THORNES, J.B. (1983): «Evolutionary Geomorphology». Geography, 68, 225-235.

TSCHANG, H. (1974): «Geomorphological observations on the tafoni forms of Hong Kong». Chung Chi Journal, 13 (1), 32-51.

TRICART, J. (Dir.) (1952): «Problèmes géomorphologiques corses». Revue de Géomorphologie Dynamique, III (4), 157-198.

TRICART, J. (1965) : Principes et méthodes de la géomorphologie. Paris. Masson. 496 pp.

TRICART, J. y CAILLEUX, A. (1965): Traité de Géomorphologie (I) Introduction à la Géomorphologie climatique. Paris. SEDES. 307 pp. 
TRICART, J. y CAILLEUX, A. (1969) : Traité de Géomorphologie (IV) Le modelé des regions séches. Paris. SEDES. 472 pp.

TURKINGTON, A.V. (1998): «Cavernous weathering in sandstone; lessons to be learned from natural exposure». The Quaterly Journal of Engineering Geology, 31(4), 375-383.

TURKINGTON, A.V. y PHILLIPS, J.D. (2004): «Cavernous weathering, dynamical instability and self-organization». Earth Surface Process and Landforms, 29, 665-675.

TWIDALE, C.R. (1982): Granite landforms. Amsterdam. Elsevier. 372 pp.

TWIDALE, C.R. (2002): «The two-stage concept of landform and landscape development involving etching: origin, development and implications of an idea». Earth-Science Reviews, 57, 37-74.

TWIDALE, C.R. y VIDAL ROMANI, J.R. (2005): Landforms and Geology of Granite terrains. Amsterdam. Balkema. 352 pp.

UGARTE, F.M. (1983): «Microformas de desagregación granular en las areniscas paleoeocenas de Algorta (Vizcaya)». Lurralde, 6, 119-125.

VIDAL ROMANI, J.R. (1985): El cuaternario en la provincia de A Coruña. Geomorfología granítica. Modelos elásticos de formación de cavidades. Tesis Doctoral. Madrid. Publicaciones de la Universidad Complutense. 283 pp.

VIDAL ROMANI, J.R. (1989): «Geomorfología granítica en Galicia». Cadernos do Laboratorio Xeolóxico de Laxe, 13, 89-163.

VIDAL ROMANI, J.R., GRAJAL, M., VILAPLANA, J.M., RODRIGUEZ, R., MACIAS, F., FERNANDEZ, S. y HERNANDEZ-PACHECO, A. (1979): «Micromodelado en el granito de Monte Louro, Galicia, España». Actas de la IV Reunión del GETC, 246-266.

VILES, H. (1988): Biogeomorphology. Oxford. Blackwell. 365 pp.

VILES, H. (2005): «Self-organized or disorganized? Towards a general explanation of cavernous weathering». Earth Surface Processes and Landforms, 30, 1471-1473.

VORONOV, P.S. (1958): «Geomorphology of East Antarctica». Soviet Antarctic Expedition, vol. 1, 20-24.

WAINWRIGHT, J., TURNBULL, L., IBRAHIM, T.G., LEXARTZA-ARTZA, I., THORNTON, S.F. y BRAZIER, R.E. (2011): «Linking environmental regimes, space and time: Interpretations of structural and functional connectivity». Geomorphology, 126, 387-404.

WERNER, B.T. (1999): «Complexity in Natural Landforms Patterns». Science, 284, 102104.

WILHELMY, H. (1958): Klimamorphologie der Massengesteine. Brunswick. Westermanns, $238 \mathrm{pp}$.

WILHELMY, H. (1964): «Cavernous rock surfaces (tafoni) in semiarid and arid climates». Pakistan Geographical Review, 19, 9-13.

WILLIAMS, R.B.G. y ROBINSON, D.A. (1982): «Sandstone sculptures in the Fontainebleau woods». The Geographical Magazine, 54, 572-579.

WRAY, R.A.L. (1997): «Quartzite dissolution : karst or pseudokarst ?». Cave and Karst Science, 24(2), 81-86. 